\title{
Identification and Validation of Key miRNAs and a microRNA-mRNA Regulatory Network Associated with Ulcerative Colitis
}

\author{
Shanshan Wang, ${ }^{1,2}$ Lei Shen, ${ }^{1}$ and Hesheng Luo ${ }^{1}$
}

\begin{abstract}
Ulcerative colitis (UC) is a chronic, nonspecific, intestinal inflammatory disease that involves various genes and pathways in its pathogenesis. The current study revealed the key miRNAs and potential target gene regulatory network as a model for predicting the molecular mechanism of UC. This may provide novel insights for unraveling the pathogenesis of UC. Differentially expressed miRNAs (DEMIs) and mRNAs (differentially expressed genes [DEGs]) between UC patients and normal controls were screened using the Gene Expression Omnibus database. DEMI target genes were predicted using the miRDB, miRWalk, starBase, TarBase, and TargetScan databases, and an miRNA-mRNA network was established using DEGs that altered in opposition to DEMIs. We verified the expression of key DEMIs in a rodent UC model. The miRNA-mRNA network contained 31 DEMIs and 199 DEGs, which showed enrichment in inflammatory bowel disease. We selected 2 key miRNAs and 4 hub genes. In addition, we identified six DEMIs and genes from the preliminary validation analysis in model tissues. In the pathophysiological process of UC, various genes and proteins display expression differences and complex interactions with each other. These findings provide new insights into the potential key mechanisms associated with UC development.
\end{abstract}

Keywords: ulcerative colitis, miRNA, mRNA, regulatory network, bioinformatics analysis

\section{Introduction}

$\mathbf{U}$ LCERATIVE COLITIS (UC) is a chronic, nonspecific, intestinal inflammatory disease with unclear pathogenesis (Torres et al., 2019). Intestinal infection, immunity, heredity, and other factors may lead to abnormal inflammatory processes in colonic mucosa. This results in further damage to intestinal epithelial mucosal barrier function, absence of recess structure, impaired secretion function, and a series of clinical symptoms and pathophysiological changes. At present, the treatment for active UC focuses mainly on controlling inflammation and relieving symptoms. However, patients are required to receive long-term treatment that may cause negative side effects. Early diagnosis and therapeutic intervention are important for minimizing the damage to UC patients and have the potential to significantly improve the prognosis. Therefore, novel biomarkers with high sensitivity and specificity are urgently needed to allow for early diagnosis of UC and thereby improve clinical outcomes.

microRNAs (miRNAs) are a type of highly conserved, short-length. endogenous, noncoding single-stranded RNA that do not have the potential to encode proteins, but can negatively regulate genes (Cheng et al., 2019). miRNAs restrain protein translation or messenger RNA (mRNA) degradation by binding to the $3^{\prime}$ untranslated regions ( $3^{\prime}$ UTRs) of mRNAs. Accumulating studies have revealed that miRNAs are involved in various biological functions, including cell proliferation, apoptosis, and inflammation. Enhancing our understanding of the relationships between miRNAs and target genes can help reveal detailed mechanisms and identify novel biomarkers for UC.

In this study, we identified mRNAs and miRNAs that are differentially expressed (DE) in UC using integrated analyses. Gene expression profiles in UC were acquired through the Gene Expression Omnibus (GEO) database and collectively analyzed based on bioinformatic analysis technology. Then, an miRNA-mRNA regulatory network was constructed after a comprehensive analysis. Finally, two potential miRNAs and four target mRNAs were selected, preliminarily validated by real-time quantitative polymerase chain reaction (RT-qPCR), and identified as significant predictors of UC. Our aim was to identify miRNAs with high clinical applicability for the early diagnosis and treatment of UC patients.

\footnotetext{
${ }^{1}$ Department of Gastroenterology and ${ }^{2}$ Key Laboratory of Hubei Province for Digestive System Disease, Renmin Hospital of Wuhan University, Wuhan, China.
} 


\section{Materials and Methods}

\section{Collection and inclusion criteria of studies}

The mRNA and miRNA microarray expression profile datasets were retrieved and downloaded from the GEO database, which was searched using the following key words: "RNA," "active ulcerative colitis," and "Homo sapiens" (organism). The inclusion criteria were as follows: (i) colon tissues (not cells) from adult patients with active UC, (ii) samples in the UC group that did not receive any interventions or treatments, and (iii) both UC and health control sample numbers were $\geq 5$. After screening, two research datasets on UC, GSE48957 and GSE48958, were included in our study. The two datasets were acquired by the same research group with the same research samples, all of which were obtained from colonic mucosal tissues, and were therefore selected to construct the UC miRNA-mRNA regulatory network. GSE48957 comprised miRNA data and included 10 active UC patients and 10 control patients whose probe platform was GPL14613 (Affymetrix Multispecies miRNA-2 Array). GSE48958 contained mRNA data and included eight active UC patients and seven control patients whose probe platform was GPL6244 (Affymetrix Human Gene 1.0 ST Array, transcript gene version).

\section{Data processing and differentially expressed gene/differentially expressed miRNA identification}

The raw data was downloaded from the GEO database, then data was normalized, and differentially expressed genes (DEGs) were identified using the limma package in Bioconductor (Gentleman et al., 2004) of the R language package (The R Foundation, Vienna, Austria). When we selected the DEGs and differentially expressed miRNAs (DEMIs), $p<0.05$ and $|\log 2 \mathrm{FC}|>1$ were considered as the screening threshold, where FC represents fold change. The heat maps and volcano plots of the mRNA and miRNA datasets were drawn using the gplots and pheatmap packages in $\mathrm{R}$ to visualize the expression values of genes in the different samples. The identified DEMIs were preserved for further bioinformatic analysis.

\section{Analyses of miRNA-mRNA targets}

For prediction of miRNA target genes, the principle of combining experimental verification and computational prediction was adopted. In this study, we used five miRNA target tools: miRDB (Liu and Wang, 2019; Chen and Wang, 2020), miRWalk (Dweep et al., 2011; Dweep and Gretz, 2015), starBase (Li et al., 2014), TarBase (Karagkouni et al., 2018), and TargetScan (Agarwal et al., 2015). miRNA targets were screened based on overlapping results from at least three tools and selected as the possible target genes. Then, the screened DEMI targets were compared with DEGs obtained above, and the DEGs that altered in opposition to the corresponding DEMI targets were selected as the key target genes. The miRNA-mRNA regulatory network was constructed within this range.

\section{Enrichment analysis of DEGs and DEMIs} and protein-protein interaction network construction

Disease enrichment of DEGs was carried out using the KOBAS (Xie et al., 2011) database, with $p<0.05$ as the screening condition to verify the effectiveness of screened DEGs. DEMI path analysis was carried out with miRPathDB 2.0 to verify the effectiveness of the regulation network construction. Gene ontology (GO) enrichment analysis, which is used for annotating genes, was conducted to determine significantly regulated functions, including the biological process (BP), cellular component (CC), and molecular function (MF). The Kyoto Encyclopedia of Genes and Genomes (KEGG) enrichment analysis was performed to present the systematic analysis, annotation, and visualization of gene functions. Both GO enrichment and KEGG pathway analyses were conducted using $\mathrm{R}$, with $p<0.05$ considered to be statistically significant.

\section{Protein-protein interaction network construction and hub gene identification}

To gain insights into the interactions of the target miRNA genes, a protein-protein interaction (PPI) network was constructed and analyzed with the STRING tool to reveal the molecular mechanisms underlying UC. Hub genes, defined as genes that play essential roles in the network, were identified according to the cutoff criterion of degree calculated using cytoHubba in Cytoscape (Su et al., 2014). The corresponding interactions were visualized using Cytoscape software.

\section{DEMI-hub gene network construction}

A Sankey diagram was used to visualize the DEMI regulatory relationship of hub genes. Key miRNAs were selected from this network.

\section{Experimental animals}

We obtained 6-8-week-old, adult, male C57BL/6 mice from Beijing HFK Bioscience Co., Ltd. (Beijing, China). All mice were housed in a room with controlled temperature $\left(21^{\circ} \mathrm{C} \pm 2{ }^{\circ} \mathrm{C}\right)$ and humidity $(50 \% \pm 5 \%)$ as well as a 12-h light/12-h dark cycle. The mice had access to a standard mouse diet and sterile water ad libitum and were acclimated to the laboratory conditions for 7 days before any experimental procedures. All experimental procedures were conducted in accordance with the institutional guidelines for the care and use of laboratory animals of Renmin Hospital at Wuhan University (Wuhan, China) and conformed to the regulations outlined in the National Institutes of Health Guide for Care and Use of Laboratory Animals. Experiments were performed under a project license (NO.: WDRM20191008) granted by Renmin hospital of Wuhan university.

\section{Dextran sulfate sodium-induced UC model}

Ten mice were randomly allocated into a control group or a dextran sulfate sodium (DSS) model group (five mice per group). The mice in the DSS model group received 3\% (w/v) DSS (160110; MP Biomedicals, Santa Ana, CA) for 7 days, while the controls were given DSS-free drinking water. All mice were sacrificed by cervical dislocation on day 8 and their colon tissues were harvested for subsequent analyses. 
Table 1. Primers Used for Real-Time Quantitative Polymerase Chain Reaction

\begin{tabular}{lll}
\hline Gene & \multicolumn{1}{c}{ Primer } & \multicolumn{1}{c}{ Sequence $\left(5^{\prime}{ }^{\prime} 3^{\prime}\right)$} \\
\hline miR-141 & Loop primer & GTCGTATCCAGTGCAGGGTCCGAGGTATTCGCACTGGATACGACCCATCTTT \\
miR-200a & F primer & TGCGCTAACACTGTCTGGTAAA \\
& loop primer & GTCGTATCCAGTGCAGGGTCCGAGGTATTCGCACTGGATACGACACATCGTT \\
HGF & F primer & TGCGCTAACACTGTCTGGTAAC \\
& Forward & AGGAACAGGGGCTTTACGTT \\
IRS1 & Reverse & GCTGCCTCCTTTACCAATGA \\
& Forward & CTCTACACCCGAGACGAACAC \\
SELE & Reverse & AACCTGCCAGACCTCCTTG \\
& Forward & ATGCCTCGCGCTTTCTCTC \\
PLEK & Reverse & GTAGTCCCGCTGACAGTATGC \\
& Forward & GAAGGAGGCCAGAAGTTTGC \\
& Reverse & AGGGAAGCGGAGATCATGAG \\
\hline
\end{tabular}

\section{Histopathological analyses}

A portion of the distal colon, which was fixed with $10 \%$ buffered formalin for over $24 \mathrm{~h}$, was mounted in paraffin and stained with hematoxylin and eosin (H\&E) before observation under a light microscope (Olympus BX53). The histological scoring system (Dieleman et al., 1998) was used to grade the severity of tissue damage induced by DSS.

\section{RNA extraction and $q R T-P C R$}

According to the manufacturer's instructions, the total RNA of colon tissues in both the UC and control groups was extracted using TRIzol reagent (Invitrogen, Carlsbad, CA). The primers were obtained from GenScript Biotech (Piscataway, NJ), and the sequences are presented in Table 1. SYBR Green probes and an ABI QuantStudio 6 system were used for qRT-PCR. miRNA expression was normalized against U6 expression, and mRNA expression of each target gene was normalized against GADPH expression. The data were calculated by means of the $2^{-\Delta \Delta \mathrm{Ct}}$ method. The experiment was replicated three times.

\section{Statistical analyses}

The data obtained from the experiments described above were analyzed using SPSS Statistics 23.0 software (IBM, Armonk, NY) and are presented as means \pm standard deviations (SDs). Student's $t$-test was used to assess the differences among and between groups, respectively; $p$ values $<0.05$ were considered to be statistically significant.

\section{Results}

Screening and identification of DE miRNAs and mRNAs in UC

The criteria for screening DEMIs and DEGs included $\mid \log$ $2 \mathrm{FC} \mid>1$ and $p<0.05$. Among the selected GEO datasets, 34 DEMIs, including 21 downregulated and 12 upregulated miRNAs, were found in the GSE48957 dataset (Fig. 1A, B), whereas 781 DEGs, including 515 upregulated and 266 downregulated genes, were found in the GSE48958 profile (Fig. 1C, D).

\section{miRNA-target gene interactions}

Following data preprocessing and analysis of the two datasets, the DEMI target genes were predicted using the database described above. The predictions were verified by over five algorithms, including miRDB, miRWalk, starBase, TarBase, and TargetScan. There were no effective target genes found in hsa-mir-1308, hsa-mir-1973, and hsa-mir-3172. The screened DEMI targets were compared with the DEGs, and the DEGs that altered in opposition with the corresponding DEMI targets were selected as the key target genes. We identified a total of 199 key DEGs, 138 of which were upregulated and 61 of which were downregulated. The miRNA-mRNA interaction network was visualized using Cytoscape software (Fig. 2).

\section{Functional pathway and disease enrichment analyses of key DEGs and pathway enrichment analysis of DEMIs}

The GO functional annotation analysis results indicated that for the key DEGs, the most enriched terms were "response to peptide hormone," "regulation of vasculature development," and "leukocyte migration" in the BP category; "secretory granule membrane," "collagen-containing extracellular matrix," and "membrane raft" in the CC category; and "glycosaminoglycan binding" and "secondary active transmembrane transporter activity" in the MF category (Fig. 3A). In addition, KEGG pathway analysis showed that the most enriched pathways were the PI3K-Akt signaling pathway, TNF signaling pathway, rheumatoid arthritis, and leukocyte transendothelial migration (Fig. 3B), some were conducted significantly participating in UCrelated pathways. DEG disease enrichment analysis was carried out using the KOBAS database with $p<0.05$. Among the list of the top 20 diseases (Fig. 3C), inflammatory bowel disease (IBD) showed enrichment. Therefore, we believe that the screened key DEGs are representative. DEMI pathway enrichment analysis was carried out using miRPathDB 2.0, in which the statistics are based on experimental evidence. The analysis results were visualized using heat maps (Fig. 3D). The PI3K-Akt signaling pathway and TNF signaling pathway are included in the previous pathway enrichment that DEMIs may participate in. The TNF signaling pathway has been identified as the key pathway in the mechanism research of UC, which further verifies the rationality of our miRNA-mRNA regulatory network construction. Therefore, we speculated that the PI3K-Akt signaling pathway also played a certain role in the pathogenesis of UC. 
A

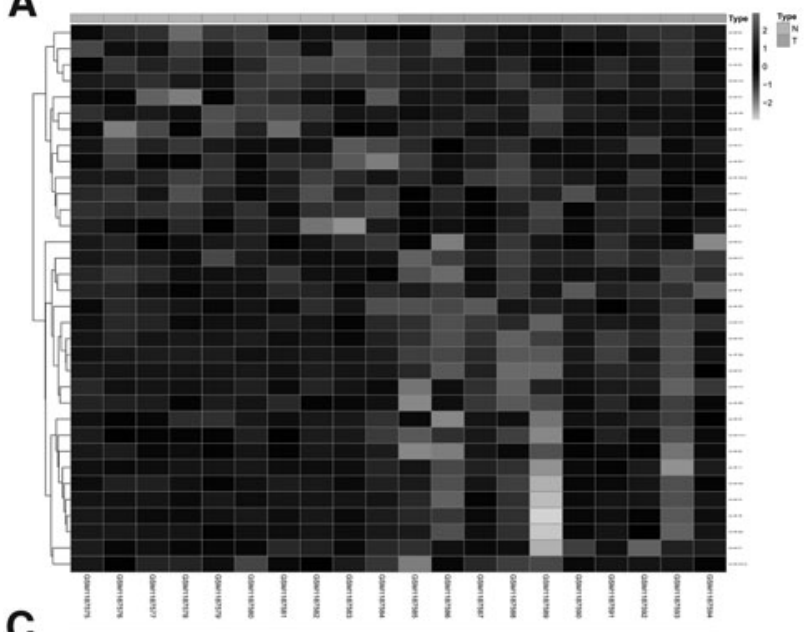

C

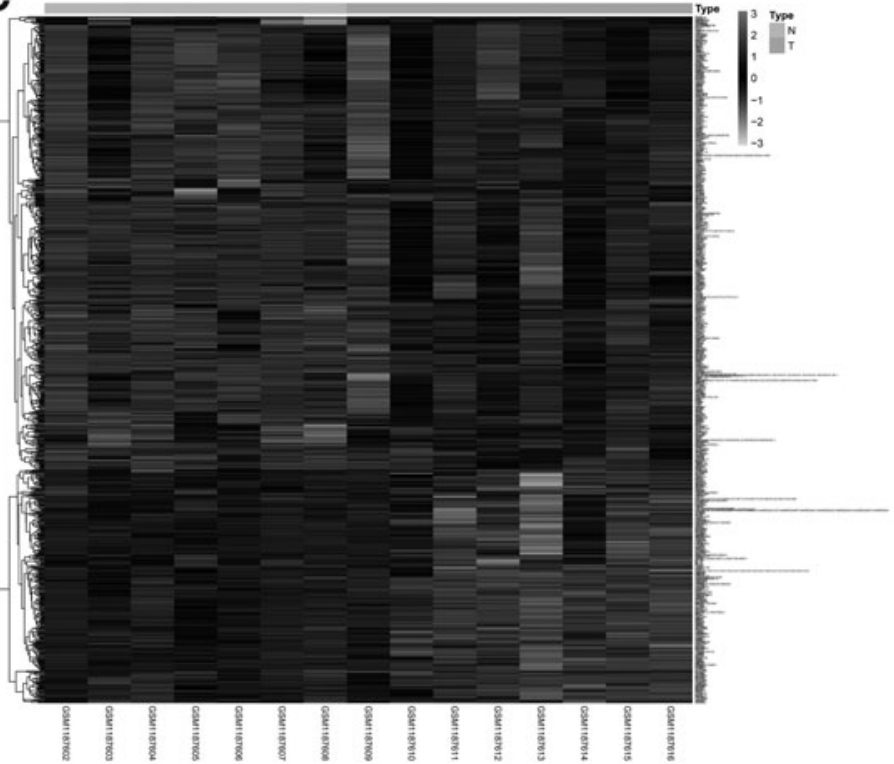

B
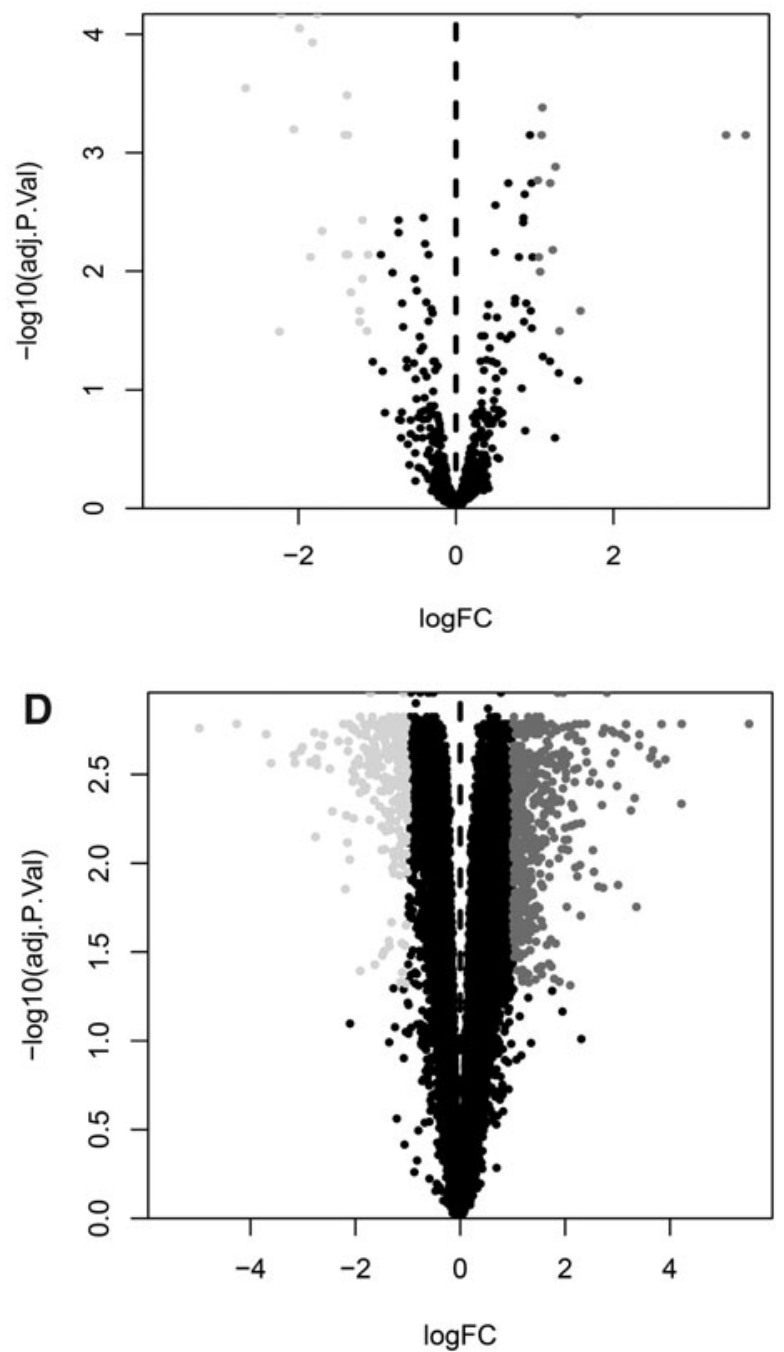

FIG. 1. Identification of DEMIs and mRNA analysis. (A) Heat map and (B) volcano plot of DEMIs in GSE48957. Darker shades of gray represent upregulated miRNAs and lighter shades of gray represent downregulated miRNAs. (C) Heat map and (D) volcano plot of DEMIs in GSE48958. Darker shades of gray represent upregulated mRNAs and lighter shades of gray represent downregulated mRNAs. DEMIs, differentially expressed miRNAs. For ease of reading, text can be magnified online.

\section{Construction of key DEG-PPI}

To identify the hub genes among key DEGs, PPI networks were constructed using the STRING database and Cytoscape software. With degree as the criterion, the network comprised 158 nodes and 483 edges (Fig. 4A). Highly connected proteins in a network are key mechanisms for regulation and defined as hub proteins ( $\mathrm{Yu}$ et al., 2017). Among them, FN1 (degree=42), CD44 (degree=37), PTGS2 (degree $=25)$, SELL $($ degree $=23)$, and CXCL1 (degree $=22$ ) and other genes were at the core of the network. The top 20 hub genes were identified by evaluating degrees with the cytoHubba plugin in Cytoscape (Fig. 4B).

\section{Construction of the DEMI-hub gene network and selection of potential genes}

Subsequently, a DEMI-hub gene network was constructed using the $\mathrm{R}$ language package (Fig. 4C). DEMIs targeting three or more hub genes, including miR-10b, miR-141, miR192, miR-194, miR-200a, miR-200b, miR-429, and miR548a-3p, were considered to be key miRNAs. In the network, miR-141 and miR-200a have the highest degree of connectivity and both target HGF, IRS1, SELE, and PLEK. Therefore, we speculated that they are potential key miRNAs and target genes.

\section{Verification of key miRNAs and target genes by $R T-q P C R$}

We demonstrated the identified key miRNAs in the colon tissues of rodent UC models. H\&E staining of DSS-induced UC and control samples (Fig. 5A, B) implied successful model preparation. RT-qPCR results indicated that the miRNAs and target genes were significantly expressed in UC tissues compared with controls. We observed that two miRNAs and four target genes were DE in UC samples 

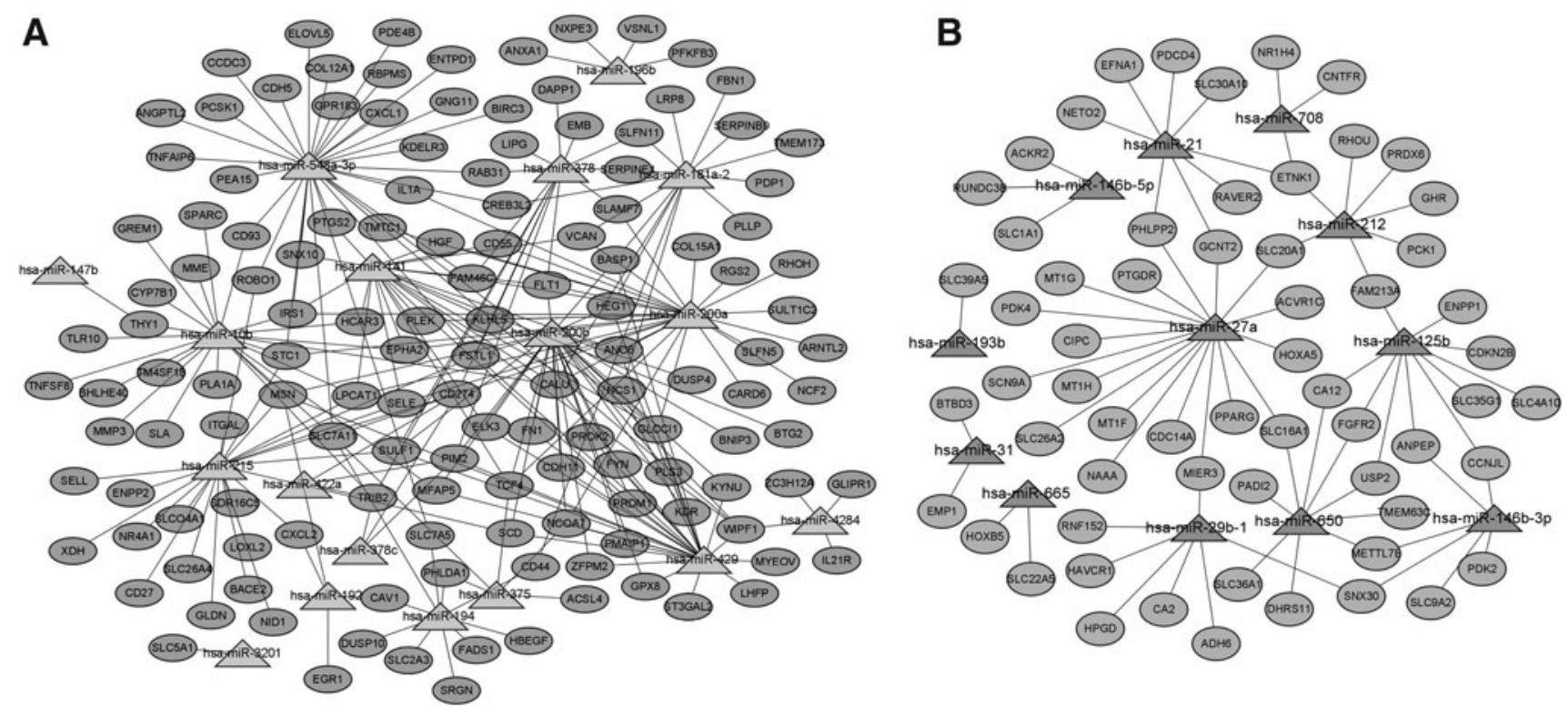

FIG. 2. DEMI-target DEM interactions. (A) The down-regulated miRNA regulatory network. (B) The up-regulated miRNA network. Interaction networks of DEMIs and target DEGs in UC. The triangle represents miRNAs and the ellipse represents mRNAs. Darker shades of gray represents upregulated genes and lighter shades of gray represents downregulated genes.

(Fig. 5C-H). Two miRNAs were downregulated and four target genes were upregulated, which were consistent with results of the microarray analysis.

\section{Discussion}

The biogenesis of UC is an extremely complex process during which many genetic and epigenetic modifications of driving genes occur. Analysis of the UC mechanism at the gene transcription level provides a new basis and direction for molecular biological research on UC as well as opens up new ideas for improving the accuracy of UC diagnosis and targeted treatment. miRNAs are critical functional molecules in UC (Xu and Zhang, 2016) and have consequently attracted significant interest as potential therapeutic targets (Schaefer et al., 2015; Lin et al., 2016). However, because there are often numerous miRNAs that influence inflammatory processes, selecting the most suitable target for UC diagnosis and treatment remains a significant challenge. In this study, the miRNA-mRNA regulatory network with differential gene expression levels in UC was constructed using bioinformatic analyses, which provide evidence that these genes and miRNAs may play various roles in development of active UC. We finally verified that miR-141, miR-200a, and their target genes, HGF, IRS1, SELE, and $P L E K$, were DE in UC, which meant that these genes could regulate the cellular process and may be represented as candidate biomarkers for UC.

In the present study, we analyzed the differential expression levels of miRNA and mRNA in the colonic mucosal tissues of UC patients using GEO datasets, GSE48957 and GSE48958, and 34 DEMIs and 781 DEGs were screened out. We further predicted the DEMI target genes and intersections with DEGs, and 31 DEMIs and 199 key DEGs were ultimately obtained.

The biological functions that DEGs are involved in may indicate the overall pathological characteristics of UC.
KEGG pathway enrichment demonstrated that PI3K-Akt and TNF signaling pathways may participate in BP regulation in UC, which was similar to the DEMI pathway enrichment process mentioned previously. The TNF signaling pathway has been identified as the key pathway in the mechanism research of UC, and TNF- $\alpha$ is one of the common proinflammatory cytokines. It can promote the activation and aggregation of local inflammatory cells as well as directly participate in nonspecific inflammatory responses, which have been common indicators for evaluating UC inflammation levels (Xiao et al., 2013). The PI3K/Akt signaling pathway has been shown to be associated with the incidence of UC, p-Akt expression showed significant increases in the colonic mucosa of the DSS-induced rat model and mediated the occurrence and progression of UC [Miao et al., 2015]. In addition, overactivation of the PI3K/Akt signaling pathway can also induce UC-related cancerization in an inflammatory environment [Khare et al., 2015].

The constructed PPI networks of key DEGs revealed direct or indirect interactions between most of the proteins. The top 20 DEGs were selected as hub genes using the cytoHubba plugin of Cytoscape. Among the hub genes that we identified, FNI was highlighted as having the highest degree of connectivity. FN1 (Lai and Tay 2016), an important cellular matrix protein located at the core, is involved in encoding fibulin and can induce the expression of specific matrix metalloproteinases as well as promote the invasion and metastasis of tumors. Furthermore, a large amount of extracellular matrix deposition may lead to organ fibrosis (Rai et al., 2017), which provides a research basis for the study of intestinal wall fibrosis in UC patients.

DEMIs target hub genes and are believed to be the key miRNAs in UC. A total of eight DEMIs targeted three or more hub genes, including miR-10b, miR-141, miR-192, miR-194, miR-200a, miR-200b, miR-429, and miR-548a$3 p$. Moreover, among the eight miRNAs that might exert effects on the development of UC, miR-192 has been 


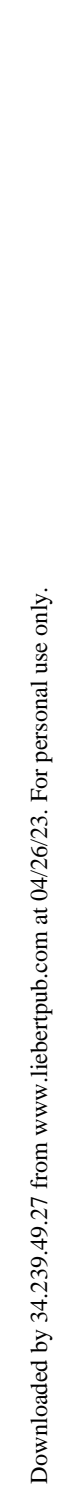

$m$
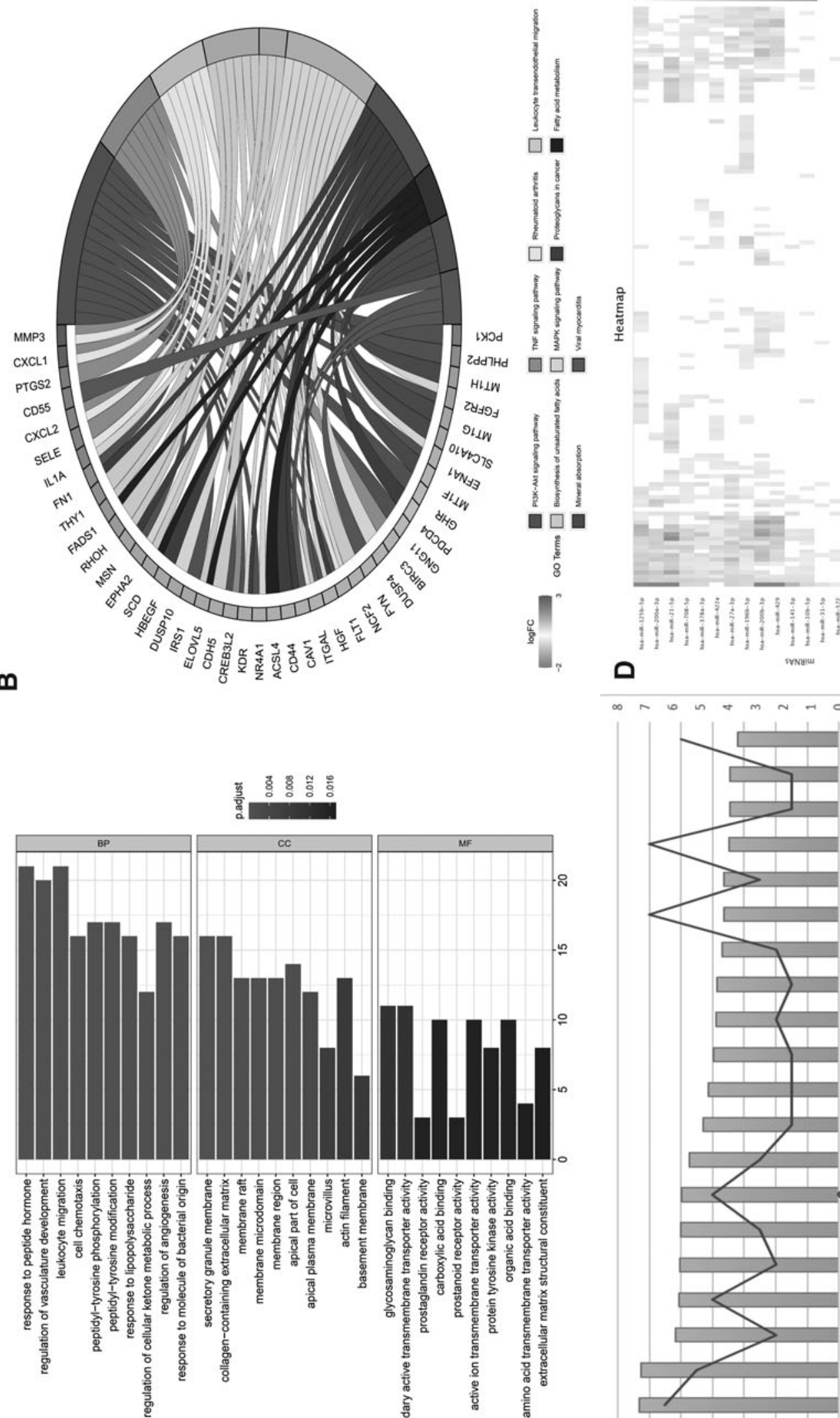

$\varangle$

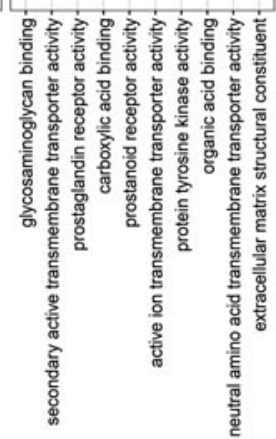

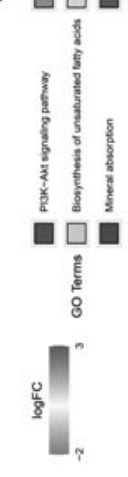

口

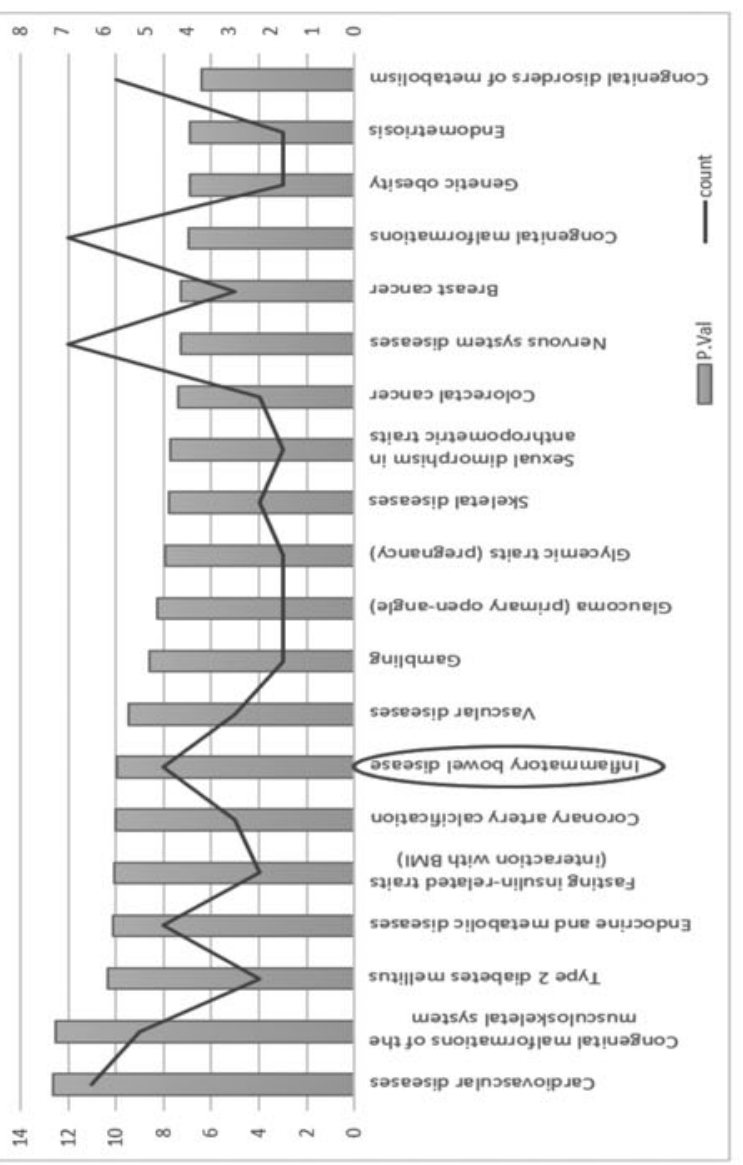

总要

,

욜

突

క.

要

ग

娄青

椀

过

क

可

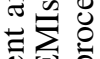

핀

氜㻤

허느응

ช 근응

Ð

๑ $\Xi \dot{\Xi}$

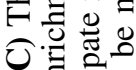

르ㅇㅝㅠ

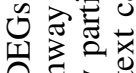

穴至

入芯茪 oi

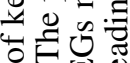
이민 हิอิ

Ð.

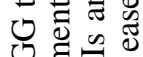

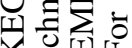

님

ฮ류

그워

至 럴

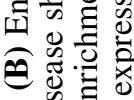

잉흘

핌 预.

两定

区ै

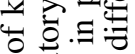

噶

○品.茫

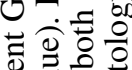

ฮั气

을

至茯空

ङ.

- 节

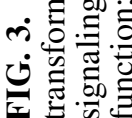


A

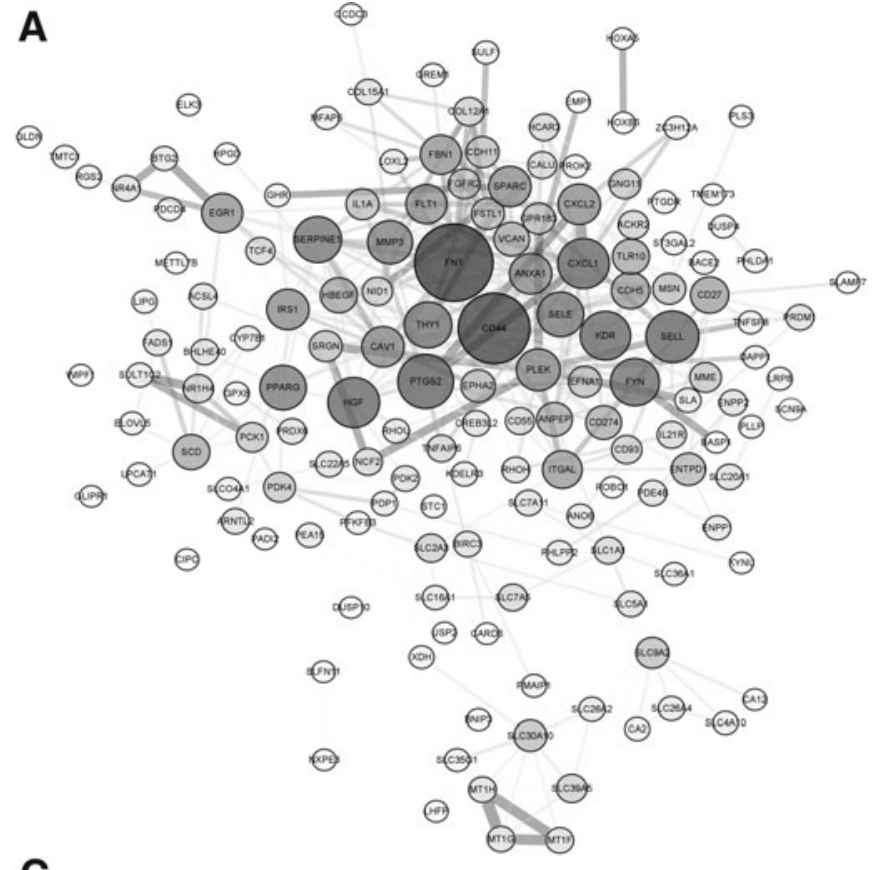

B

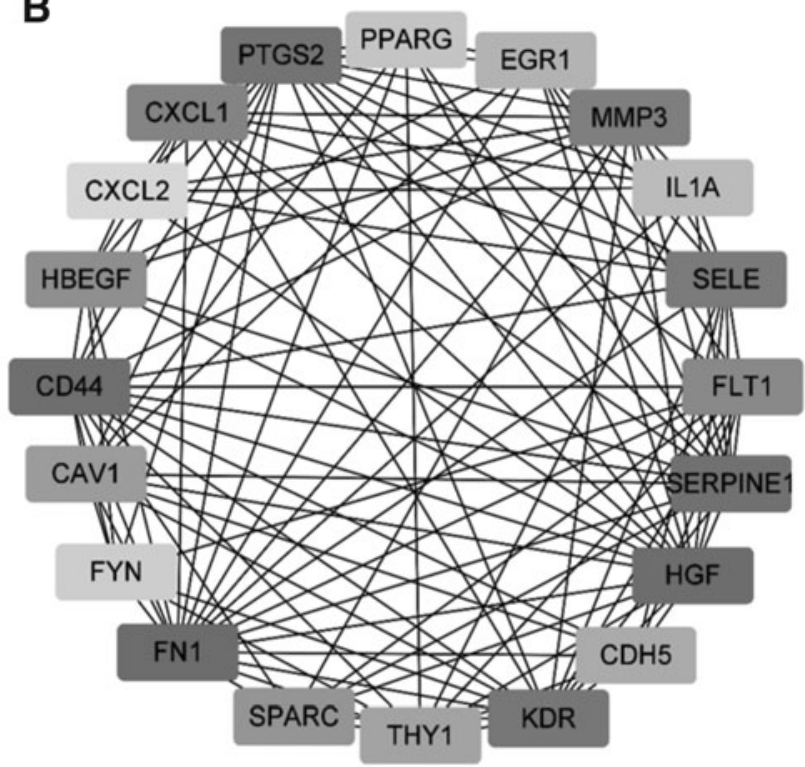

C
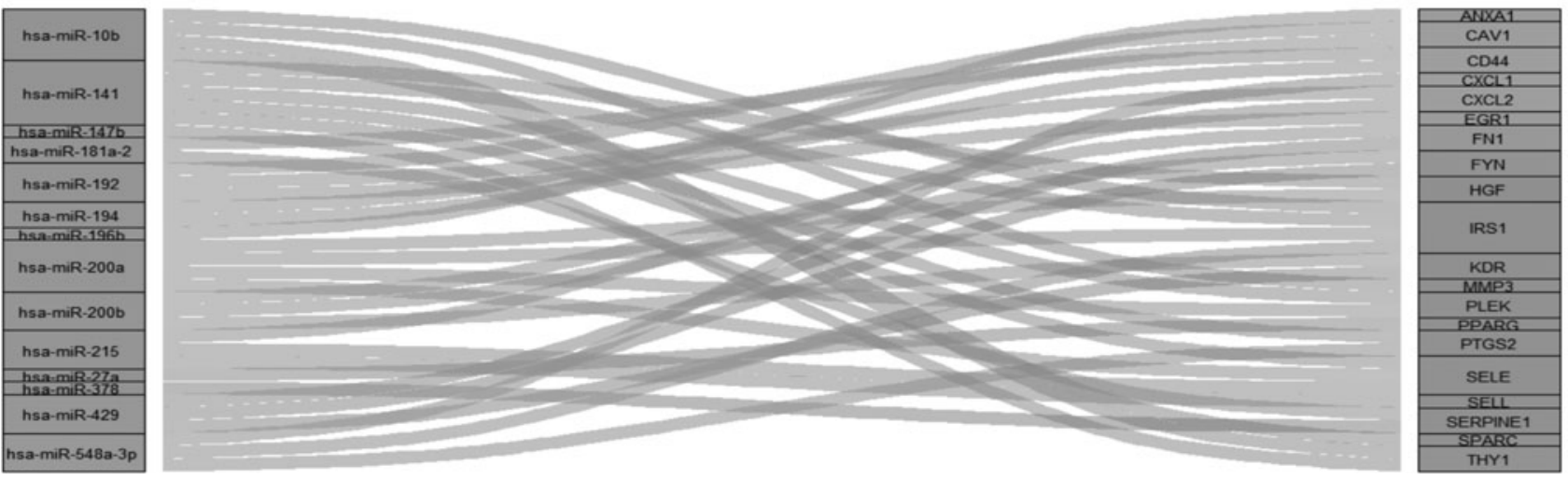

FIG. 4. (A) PPI networks of key DEGs. (B) The interaction of 20 hub genes. (C) DEMI-hub gene networks by Sankey diagram. PPI, protein-protein interaction.

identified in mechanistic studies as a biomarker for UC. miR-192, which is mainly expressed in colonic mucous epithelial cells, has been shown to be decreased in patients with active UC (Wu et al., 2008; Konstantinidis et al., 2020). miR-192 regulates the expression levels of several chemokines, including nucleotide-binding oligomerization domaincontaining protein 2 , interleukin 8 , and $\mathrm{C}-\mathrm{X}-\mathrm{C}$ motif ligand 3 , in colonic mucosa as well as the phosphorylation of nuclear factor kappa B (Belcheva, 2017). Given these studies and our study, miR-192 may be a key miRNA in regulating the innate immune response in intestinal epithelial cells and the pathogenesis of UC, hence its low expression in the colonic mucosa of UC patients. miR-200b (Chen et al., 2013) has also been shown to be decreased in the inflammatory mucosa of IBD patients, displaying a positive correlation with the expression of E-cadherin, but a negative one with the levels of transforming growth factor beta 1 and vimentin. In another study, miR-200b was demonstrated to partially protect intestinal epithelial cells from fibrogenesis by suppressing the epithelial-mesenchymal transition in vitro
(Chen et al., 2012). In summary, miR-200b appears to play a potential role in improving the pathophysiology and clinical outcomes of IBD. The remaining key DEMIs have not been systematically studied in terms of UC.

Of note, miR-141 and miR-200a have the highest degree of connectivity in the network and both target HGF, IRSI, SELE, and PLEK, which suggested that hsa-miR-141 and hsa-miR-200a may be key regulators of HGF, IRS1, PLEK, and SELE. It was shown that both patients with active UC and mice during the acute inflammatory phase of DSSinduced colitis have increased mucosal and serum expression of $H G F$ that positively correlated with a neutrophil enrichment score, which might be a surrogate marker of colonic inflammation (Stakenborg et al., 2020). HGF could regulate the proliferation and movement of intestinal epithelial cells (Tahara et al., 2003). Other genes have not been systematically studied in UC, and more studies are needed to clarify their mechanism in UC. We identified that two miRNAs and four target mRNAs selected above were DE in the DSS-induced mouse model. The results were in line with 

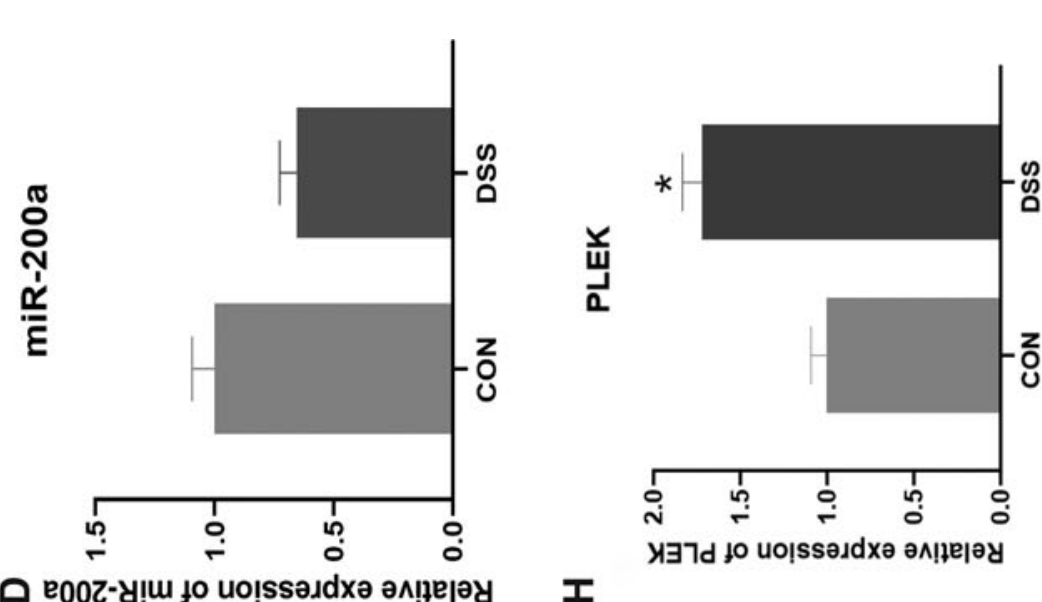

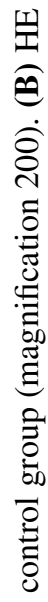

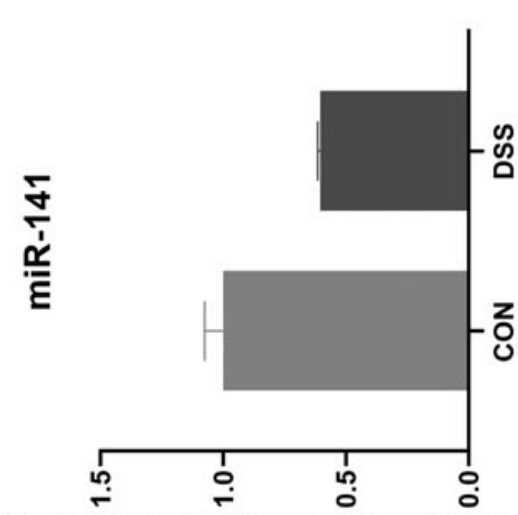

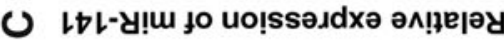

ज

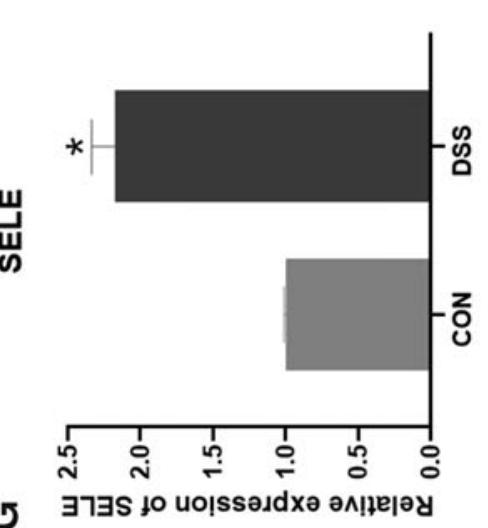

$\Xi$

$\exists \Xi$

画

可

可牙灵

苞雪

$\dot{j} \dot{x}$

$\Xi \approx$

둘

侊望:

氜氠
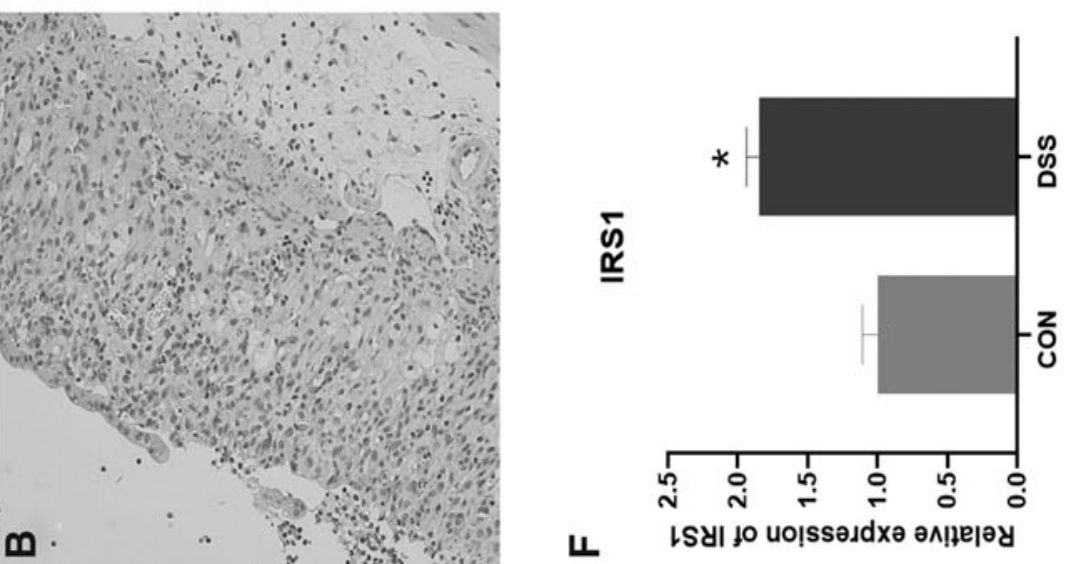

究

율

i 뜬

릴

응

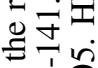

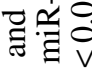

.0

官的

ปี

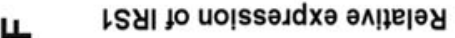
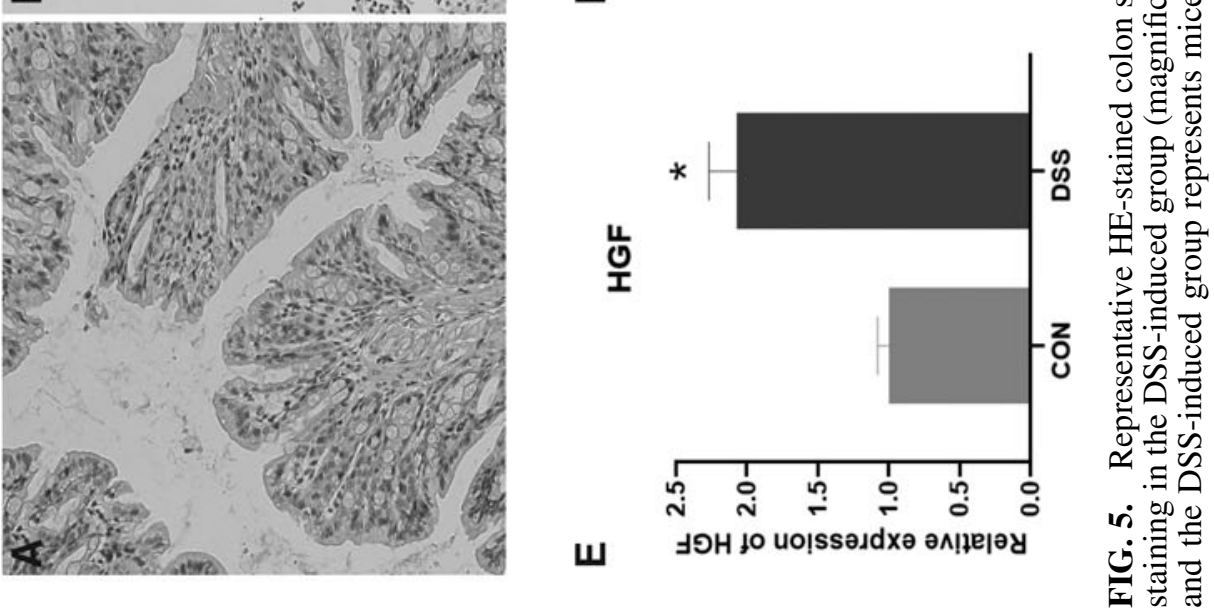
the microarray analysis. Therefore, we speculated that these miRNAs and their target mRNAs may play an important role in UC development. By the implication of KEGG enrichment, it was suggested that $H G F$ and IRSI are closely related to the PI3K-Akt signaling pathway. We inferred that $H G F$ and IRSI, which were regulated by miR-141 and miR200a, may play vital roles in development of UC in the PI3K-Akt signaling pathway, which can provide a new perspective for future studies.

In our study, bioinformatic analysis was used to comprehensively analyze miRNA and mRNA expression changes in the pathophysiological process of UC. We constructed the miRNA-mRNA regulation network in the process of UC and screened for key DEGs. Meanwhile, 2 key mRNAs and 4 target genes were identified and preliminarily confirmed in the aforementioned mouse model, highlighting their potential role as biomarkers related to UC.

There are several limitations to our study. The number of samples that we obtained from the GEO datasets was small, thereby generating some bias during gene analysis. In addition, the functions and molecular mechanisms of genes are very complicated, and predictions based only on bioinformatics require cellular and animal experiments for verification.

\section{Conclusions}

In summary, this study provides a theoretical basis and new ideas for future screening of miRNA and its target genes for more accurate UC diagnosis and treatment. However, further experimental and functional studies are warranted to determine the exact processes and mechanisms of UC.

\section{Authors' Contributions}

S.W., L.S., and H.L. performed the experiments; S.W. and L.S. analyzed the data, prepared the figures, and drafted the manuscript; H.L. edited and revised the manuscript; and S.W. is responsible for conception and design of the research. Dr. H.L. confirmed that all authors have contributed to and agreed on the content of the manuscript.

\section{Disclaimer}

This research did not receive any specific grant from funding agencies in the public, commercial, or not-for-profit sectors.

\section{Disclosure Statement}

No competing financial interests exist.

\section{Funding Information}

This research did not receive any specific grant from funding agencies in the public, commercial, or not-for-profit sectors.

\section{References}

Agarwal, V., Bell, G.W., Nam, J.W., and Bartel, D.P. (2015). Predicting effective microRNA target sites in mammalian mRNAs. Elife 4, e05005.

Belcheva, A. (2017). MicroRNAs at the epicenter of intestinal homeostasis. Bioessays 39, 3.

Cheng, M., Yang, J., Zhao, X., Zhang, E., Zeng, Q., Yu, Y., et al. (2019). Circulating myocardial microRNAs from in- farcted hearts are carried in exosomes and mobilise bone marrow progenitor cells. Nat Commun 10, 959.

Chen, Y., Ge, W., Xu, L., Qu, C., Zhu, M., Zhang, W., et al. (2012). miR-200b is involved in intestinal fibrosis of Crohn's disease. Int J Mol Med 29, 601-606.

Chen, Y., and Wang, X. (2020). miRDB: an online database for prediction of functional microRNA targets. Nucleic Acids Res 48(D1), D127-D131.

Chen, Y., Xiao, Y., Ge, W., Zhou, K., Wen, J., Yan, W., et al. (2013). miR-200b inhibits TGF-beta1-induced epithelialmesenchymal transition and promotes growth of intestinal epithelial cells. Cell Death Dis 4, e541.

Dieleman, L.A., Palmen, M.J., Akol, H., Bloemena, E., Peña, A.S., Meuwissen, S.G., et al. (1998). Chronic experimental colitis induced by dextran sulphate sodium (DSS) is characterized by Th1 and Th2 cytokines. Clin Exp Immunol 114, 385-391.

Dweep, H., and Gretz, N. (2015). miRWalk2.0: a comprehensive atlas of microRNA-target interactions. Nat Methods 12, 697.

Dweep, H., Sticht, C., Pandey, P., and Gretz, N. (2011). miRWalk - database: prediction of possible miRNA binding sites by "walking" the genes of three genomes. J Biomed Inform 44, 839-847.

Gentleman, R.C., Carey, V.J., Bates, D.M., Bolstad, B., Dettling, M., Dudoit, S., et al. (2004). Bioconductor: open software development for computational biology and bioinformatics. Genome Biol 5, R80.

Karagkouni, D., Paraskevopoulou, M.D., Chatzopoulos, S., Vlachos, I.S., Tastsoglou, S., Kanellos, I., et al. (2018). DIANA-TarBase v8: a decade-long collection of experimentally supported miRNA-gene interactions. Nucleic Acids Res 46(D1), D239-D245.

Khare, V., Dammann, K., Asboth, M., Krnjic, A., Jambrich, M., and Gasche, C. (2015). Overexpression of PAK1 promotes cell survival in inflammatory bowel diseases and colitisassociated cancer. Inflamm Bowel Dis 21, 287-296.

Konstanitidis, A.O., Pardali, D., Adamama-Moritou, K.K., Gazouli, M., Dovas, C.I., Legaki, E., et al. (2020). Colonic mucosal and serum expression of microRNAs in canine large intestinal inflammatory bowel disease. BMC Vet Res 16, 69.

Lai, C.J., and Tay, B.H. (2016). Pharmacophore-based screening targeted at upregulated FN1, MMP-9, APP reveals therapeutic compounds for nasopharyngeal carcinoma. Comput Biol Med 69, 158-165.

Li, J.H., Liu, S., Zhou, H., Qu, L.H., and Yang, J.H. (2014). starBase v2.0: decoding miRNA-ceRNA, miRNA-ncRNA and protein-RNA interaction networks from large-scale CLIP-Seq data. Nucleic Acids Res 42(Database issue), D92-D97.

Lin, J., Zhang, X., Zhao, Z., Welker, N.C., Li, Y., Liu, Y., et al. (2016). Novel MicroRNA Signature to Differentiate Ulcerative Colitis from Crohn Disease: a Genome-Wide Study Using Next Generation Sequencing. Microrna 5, 222-229.

Liu, W., and Wang, X. (2019). Prediction of functional microRNA targets by integrative modeling of microRNA binding and target expression data. Genome Biol 20, 18 .

Miao, X., Tang, Q., Miao, X., Wu, Y., Qian, J., Zhao, W., et al. (2015). ErbB3 binding protein 1 (EBP1) participates in the regulation of intestinal inflammation via mediating Akt signaling pathway. Mol Immunol 67(2 Pt B), 540-551.

Rai, R., Verma, S.K., Kim, D., Ramirez, V., Lux, E., Li, C., et al. (2017). A novel acetyltransferase p300 inhibitor ameliorates hypertension-associated cardio-renal fibrosis. Epigenetics 12, 1004-1013. 
Schaefer, J.S., Attumi, T., Opekun, A.R., Abraham, B., Hou, J., Shelby, H., et al. (2015). MicroRNA signatures differentiate Crohn's disease from ulcerative colitis. BMC Immunol 16, 5.

Stakenborg, M., Verstockt, B., Meroni, E., Goverse, G., De Simone, V., Verstockt, S., et al. (2020). Neutrophilic HGFMET signaling exacerbates intestinal inflammation. J Crohns Colitis 17, jjaa121.

Su, G., Morris, J.H., Demchak, B., and Bader, G.D. (2014). Biological network exploration with Cytoscape 3. Curr Protoc Bioinformatics 47, 8.13.1-24.

Tahara, Y., Ido, A., Yamamoto, S., Miyata, Y., Uto, H., Hori, T., et al. (2003). Hepatocyte growth factor facilitates colonic mucosal repair in experimental ulcerative colitis in rats. J Pharmacol Exp Ther 307, 146-151.

Torres, P., Cañete, F., Núñez, L., Aguilar, A., Mesonero, F., Calafat, F., et al. (2019). Spacing the administration interval of Anti-TNF Agents: a valid strategy for patients with inflammatory bowel disease? Dig Dis Sci 65, 2036-2043.

Wu, F., Zikusoka, M., Trindade, A., Dassopoulos, T., Harris, M.L., Bayless, T.M., et al. (2008). MicroRNAs are differentially expressed in ulcerative colitis and alter expression of macrophage inflammatory peptide- 2 alpha. Gastroenterology 135, 1624-1635.

Xiao, B., Laroui, H., Ayyadurai, S., Viennois, E., Charania, M.A.,Zhang, Y., et al. (2013). Mannosylated bioreducible nanoparticle-mediated macrophage-specific TNF-alpha RNA interference for IBD therapy. Biomaterials 34, 7471-7482.
Xie, C., Mao, X., Huang, J., Ding, Y., Wu, J., Dong, S., et al. (2011). KOBAS 2.0: a web server for annotation and identification of enriched pathways and diseases. Nucleic Acids Res 39(Web Server issue), W316-W322.

$\mathrm{Xu}, \mathrm{X.M.}$, and Zhang, H.J. (2016). miRNAs as new molecular insights into inflammatory bowel disease: crucial regulators in autoimmunity and inflammation. World J Gastroenterol 22, 2206-2218.

Yu, D., Lim, J., Wang, X., Liang, F., and Xiao, G. (2017). Enhanced construction of gene regulatory networks using hub gene information. BMC Bioinformatics 18, 186.

Address correspondence to:

Hesheng Luo, MD

Department of Gastroenterology Renmin Hospital of Wuhan University Hubei Zhang Road (formerly Ziyang Road) Wuchang District No. 99, Jiefang Road 238 Wuhan 430060

China

E-mail: 1hswhdxrmyy@126.com

Received for publication September 11, 2020; received in revised form November 4, 2020; accepted November 14, 2020. 